\title{
Discapacidad Laboral por Dolor Lumbar. Estudio Caso Control en Santiago de Chile
}

\author{
WORK DISABILITY FOR LOWER BACK PAIN. CASE CONTROL STUDY IN SANTIAGO
}

Claudio Muñoz Poblete'1, Sergio Muñoz Navarro', Jairo Vanegas López ${ }^{2}$

1. Depto. Salud Pública, Universidad de La Frontera, Chile.

2. Facultad de Ciencias Médicas, Universidad Santiago de Chile.

\section{RESUMEN}

Antecedentes. La discapacidad laboral por dolor lumbar causa ausencia laboral y gastos económicos. Se ha responsabilizado su presencia a condiciones del entorno laboral y a la manipulación manual de cargas (MMC). Se evaluó la asociación entre niveles de exposición laboral a MMC y presencia de discapacidad en trabajadores protegidos por ley laboral en Santiago de Chile.

Material y Método. Estudio Caso Control. Exposición y covariables de interés fueron medidas en puesto de trabajo. Los análisis incluyeron Modelos de Regresión Logística Múltiple.

Resultados. La exposición a MMC moderada muestra OR: 1,62 (IC95\%:0,61-4,33), la exposición a MMC alta y muy alta muestra OR de 2,75 (IC95\%:1,08-6,95).

Discusión. Existe una importante magnitud y gradiente de asociación entre MMC y discapacidad. Variables psicosociales, organizacionales e individuales también explican el fenómeno. Se sugiere rediseñar las estrategias nacionales para prevenir riesgos laborales relacionados con discapacidad por dolor lumbar.

(Muñoz C, Muñoz S, Vanegas J, 2015. Discapacidad Laboral por Dolor Lumbar. Estudio Caso Control en Santiago de Chile. Cienc Trab. SepDic; 17 [54]: 193-201).

Palabras claves: SALUD OCUPACIONAL, DOLOR LUMBAR, FACTOR DE RIESGO, DISCAPACIDAD LABORAL.

\section{ABSTRACT}

Background: Work disability due to low back pain causes lost productivity and economic expense. It has been blamed on the working environment and the manual handling of loads (MHL). The link between levels of work exposure to MHL and the disability of workers protected by labor law was evaluated in Santiago, Chile.

Materials and Method: Case-control study. Exposure and study covariables were measured in the workplace. The analyses included multiple logistic regression models.

Result and Discussion: Exposure to moderate MHL shows an OR: 1.62 (95\% CI: 0.61-4.33), high and very high exposure to MHL shows an OR of 2.75 (95\% CI: 1.08-6.95), controlled by psychosocial, organizational and individual aspects. There is a significant magnitude and gradient of association between MHL and disability. Psychosocial, organizational and individual variables also explain the phenomenon. It is suggested that national strategies be redesigned to prevent the occupational risks related to disability due to low back pain.

Keywords: OCCUPATIONAL HEALTH, LOW BACK PAIN, RISK FACTOR, SICK LEAVE.

\section{ANTECEDENTES}

Los trastornos musculoesqueléticos representan un 59\% de todas las enfermedades profesionales registradas por las estadísticas europeas y principal causa de ausentismo laboral de más de tres días, sin que se apliquen medidas de prevención, protección y control adecuadas. ${ }^{1}$

Correspondencia / Correspondence:

Claudio Muñoz Poblete, PhD

Depto. Salud Pública, Facultad de Medicina

Universidad de La Frontera

Claro Solar 115, Temuco, Chile

Tel.: 56-45-2596582

e-mail: claudio.munoz@ufrontera.cl

Recibido: 29 de Septiembre / Aceptado: 13 de Noviembre

Entre los trastornos musculoesqueléticos descritos en la literatura, el dolor lumbar representa uno de los principales, a pesar de los esfuerzos por controlarlo su frecuencia se ha mantenido relativamente estable. ${ }^{2,3}$ Representa, por tanto, un importante problema de salud pública debido al alto impacto en la funcionalidad de la persona, discapacidad laboral y altos costos económicos asociados. $^{4,5}$

Actualmente, la discapacidad por dolor lumbar se entiende bajo un modelo biosicosocial y una contribución causal múltiple ${ }^{6}$; sin embargo, no hay consenso científico de la contribución específica de cada componente. ${ }^{7}$

En relación a la exposición a cargas externas, no hay duda de que maniobras de levantamiento y traslado de cargas en el lugar de trabajo han sido vinculados como una causa común de dolor lumbar y de discapacidad. Sin embargo, las últimas revisiones sistemáticas disponibles no han podido establecer con certeza una relación causal en el contexto laboral..$^{7-11}$

Se hace necesario, por tanto, ejecutar estudios que permitan cuantificar de manera más precisa los riesgos a los que se encuentran 
expuestos los trabajadores, particularmente en Chile donde la insuficiente evidencia existente ${ }^{12}$ apoya la relevancia de desarrollar investigaciones que permitan comprender en nuestro contexto sociocultural un problema de gran magnitud. Dado lo anterior, este estudio evaluó la asociación entre niveles de exposición a manipulación manual de cargas (MMC) en el trabajo y la presencia de discapacidad laboral por dolor lumbar en trabajadores de empresas adheridas a una mutualidad de seguridad de Santiago de Chile.

\section{MATERIAL Y MÉTODO}

\section{Participantes}

El universo está constituido por trabajadores que realizan actividades laborales de manipulación manual de cargas como actividad fundamental en sus empresas respectivas, correspondiendo aproximadamente a 125.000 individuos de empresas del rubro manufacturero y logístico, afiliadas a una mutualidad de seguridad de Santiago de Chile.

Tomando en cuenta un nivel de significación de un 5\% y un poder estadístico de $80 \%$, se estimó un requerimiento de 302 sujetos (151 por grupo).

Los casos se obtuvieron de manera prospectiva durante un año y fueron identificados como aquellos trabajadores a quienes se les indicó reposo laboral por dolor lumbar de al menos un día, lo que definía la condición de discapacidad laboral. La indicación de reposo laboral es posterior a la evaluación médica en las cuatro agencias de salud de la mutualidad de seguridad. En segundo lugar, y posterior a la autorización de la empresa de donde provenía el caso, se accedió a los registros de la totalidad de los trabajadores expuestos a MMC, a partir de lo cual se realizó un muestreo probabilístico sin reemplazo, obteniendo un trabajador que servía de control. El control corresponde a un trabajador de una empresa que se encuentra adherida a una mutualidad de la región Metropolitana, quien, cumpliendo los criterios de inclusión, no haya presentado reposo laboral por dolor lumbar en el último año.

La muestra constituida por los casos y los controles quedó constituida por trabajadores varones que realizaban manipulación manual de cargas como actividad laboral regular, entre 18 y 65 años, relación contractual formal de al menos 35 horas, antigüedad en la empresa de al menos diez meses en la misma tarea laboral. Aceptación de consentimiento informado por parte del trabajador y autorización por parte de la empresa. Entre los criterios de exclusión estaban la existencia de una lesión traumática severa o una lesión maligna en la columna, presencia de comorbilidad, tales como enfermedades psiquiátricas o enfermedades sistémicas.

El número de casos incidentes alcanzó un total de 610, quienes presentaron indicación de reposo laboral de al menos un día. Un total de 156 sujetos cumplieron los criterios de inclusión definidos para este estudio. De los 454 trabajadores restantes, 345 correspondía a mujeres, 81 individuos tenían un contrato menor a los 10 meses y 21 quedaron excluidos por presentar lesión discal aguda compatible con hernia discal y derivados para un manejo médico especializado y 7 tenían asociado un cuadro psicológico de base. 21 trabajadores tenían una antigüedad laboral menor a diez meses o habían presentado ausencia laboral por dolor lumbar en el último año. No consintieron en participar del estudio un 5\% de los trabajadores consultados (Figura 1).
Figura 1.

Flujograma obtención de la muestra.

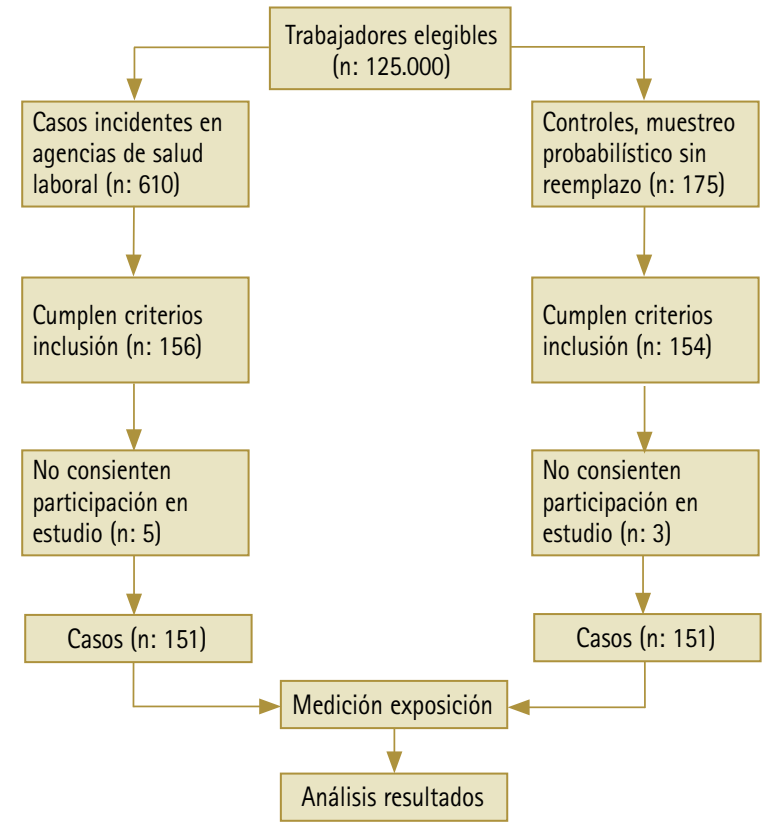

\section{Mediciones}

Discapacidad por dolor lumbar

Se utilizó información secundaria recabada de los archivos electrónicos de centros asistenciales, a través de la detección diaria de trabajadores con diagnóstico médico de lumbago e indicación médica de licencia laboral por este motivo de al menos un día.

\section{Manipulación manual de cargas}

La condición de expuesto quedó definida por las características de la manipulación manual de cargas en el trabajo, evaluada mediante la aplicación del método Manual Handling Assessment Chart (MAC) ${ }^{13}$, que permitió identificar aspectos propios del objeto manipulado, la fuerza realizada, las zonas de alcance, la postura corporal, los movimientos y aspectos propios del ambiente, con lo cual se obtuvo la clasificación de exposición baja, moderada, alta y muy alta. La aplicación de esta metodología permite obtener puntajes de corte que determinan niveles de riesgo del puesto de trabajo que en su conjunto exponen al trabajador al riesgo de desarrollar lesión lumbar. Las categorias son: bajo riesgo, moderado riesgo, alto riesgo y muy alto riesgo.

\section{Covariables}

Se aplicó el cuestionario de evaluación de riesgos psicosociales en el trabajo, SUSESO-ISTAS $21 .{ }^{14}$ Las dimensiones que engloba son exigencias psicológicas (cinco preguntas), trabajo activo y desarrollo de habilidades (cinco preguntas), apoyo social en la empresa y calidad de liderazgo (cinco preguntas), compensaciones (tres preguntas) y, finalmente, doble presencia (dos preguntas). Ofrece un puntaje que nos permite clasificar en tres niveles (bajo, moderado, alto) el riesgo para la salud debido a los factores psicosociales en el trabajo.

Mediante un cuestionario estructurado se consultó por datos sociodemográficos de interés, como nivel educacional, nivel de ingresos, información familiar; también por antecedentes de estilo de vida, tales como actividad física y hábito tabáquico. Se midió el peso y estatura como datos antropométricos relevantes; esto 
último mediante una balanza digital y un estadiómetro, con lo cual se obtuvo el índice de masa corporal (IMC). El IMC fue categorizado usando las cuatro categorías (bajo peso, normopeso, sobrepeso, obeso) propuestas por la Organización Mundial de la Salud. ${ }^{15}$

Los antecedentes laborales y factores organizacionales fueron obtenidos desde la empresa mediante la aplicación de preguntas que incluían el número de horas de trabajo semanal, antigüedad en el trabajo, número de trabajadores totales y trabajadores que comparten el puesto de trabajo, uso de faja lumbar, capacitación en manipulación manual de cargas en los últimos seis meses y horas de dedicación de profesional prevencionista.

\section{Aspectos éticos}

La propuesta de investigación y el consentimiento informado fueron evaluados y aprobados por el Comité de Ética en Investigación en Seres Humanos de la Facultad de Medicina de la Universidad de Chile.

\section{Análisis estadístico}

El proceso de análisis estadístico consideró la utilización de Stata 11.0. Se realizó una descripción de las características basales de los casos y controles de acuerdo a la variable de exposición y de los subgrupos formados por las categorias laborales, sociodemográficas y demás variables. Para los análisis estadísticos fueron generadas variables dummy. Se incluyeron pruebas de significancia estadística con el objetivo de orientarnos a identificar de manera exploratoria aquellas variables que pueden estar mediando la relación entre la MMC y la discapacidad laboral por dolor lumbar. Basado en la categorización del puntaje total que plantea el método MAC -y que permite comparar el nivel de exposición a manipulación manual de cargas-, divide la exposición en baja, moderada, alta y muy alta. El análisis principal consistió en la estimación de la asociación entre la variable MMC y la presencia de discapacidad laboral por dolor lumbar. A través de un modelo de regresión logística múltiple completo, se buscó obtener una estimación más precisa de la odd ratio (OR). Se seleccionaron las covariables de control que mejor contribuyan a la construcción de un modelo, descartando aquellas que no muestran significancia estadística

\section{RESULTADOS}

En la Tabla 1 se observa que existe una mayor concentración de casos $(66,2 \%)$ con exposición alta y muy alta al compararlos con los controles en esta condición (46,4\%). Por su parte, los trabajadores que se encuentran con exposición baja a manipulación manual de cargas alcanzan el 6,0\% para el grupo de casos y $15,2 \%$ para el grupo de controles.

Tabla 1.

Nivel de exposición a manipulación manual de cargas en el trabajo según grupo casos y grupo controles.

$\begin{array}{lcccc} & n & \text { Casos (n: 151) } & \text { Controles (n: 151) } & \text { valor p } \\ \text { MMC (\%) } & & & & <0,001 \\ \text { Exp. Baja } & 32 & 6,0(9) & 15,2(23) & \\ \text { Exp. Moderada } & 100 & 27,8(42) & 38,4(58) & \\ \text { Exp. Alta } & 115 & 45,7(100) & 30,5(46) & \\ \text { Exp. Muy alta } & 55 & 20,5(31) & 15,9(24) & \end{array}$

Test exacto Fisher, valor $p<0,05$.
Tabla 2.

Características antropométricas y estilo de vida según grupo casos y grupo controles.

\begin{tabular}{lccc} 
& Casos (n: 151) & Controles (n: 151) & valor $p$ \\
\hline Edad (media años DS) & $35,5( \pm 10,9)$ & $37,1( \pm 10,3)$ & $0,168^{*}$ \\
Altura (media cm. DS) & $171( \pm 7,07)$ & $172( \pm 6,53)$ & $0,872^{*}$ \\
Peso (media kg. DS) & $81,8( \pm 13,51)$ & $79,6( \pm 8.65)$ & $0,097^{*}$ \\
IMC (media kg/m2 DS) & $27,5( \pm 3,85)$ & $26,9( \pm 2,4)$ & $0,070^{*}$ \\
Categoria IMC (\%) & & & $<0,001$ \\
$\quad$ Normo peso & 26,5 & 19,2 & \\
$\quad$ Sobrepeso & 51,0 & 70,2 & \\
$\quad$ Obeso & 22,5 & 10,6 & \\
Fumador (\%) & & & \\
$\quad$ Nunca & 44,4 & 35,8 & \\
$\quad$ Ocasionalmente & 30,5 & 43,1 & \\
$\quad$ Frecuentemente & 25,2 & 21,2 & \\
Actividad fisica (\%) & & & \\
$\quad$ Nunca & 22,5 & 18,5 & \\
$\quad$ Rara vez & 35,8 & 36,4 & \\
$\quad$ Frecuentemente & 41,7 & 45,0 & \\
Test exacto Fisher, valor $p<0,05$ & $* t-$ test, valor $p<0,05$ & &
\end{tabular}

Tabla 3.

Características sociodemográficas según grupo casos y grupo controles.

\begin{tabular}{|c|c|c|c|}
\hline & Casos (n: 151) & Controles (n: 151) & valor $p$ \\
\hline Estado Marital (\%) & & & 0,065 \\
\hline Sin pareja & 47,0 & 37,8 & \\
\hline Con pareja & 53,0 & 62,3 & \\
\hline Número de hijos (\%) & & & 0,191 \\
\hline Sin hijos & 28,5 & 23,2 & \\
\hline 1 hijo & 23,2 & 25,2 & \\
\hline 2 hijos & 30,5 & 24,5 & \\
\hline 3 o más hijos & 17,9 & 27,2 & \\
\hline \multicolumn{2}{|c|}{ Número de personas dependientes (\%) } & & 0,062 \\
\hline 0 personas & 31,1 & 19,2 & \\
\hline 1 persona & 9,3 & 11,3 & \\
\hline 20 más personas & 59,6 & 69,5 & \\
\hline Nivel educacional (\%) & & & 0,525 \\
\hline Primaria incom./comp. & 24,5 & 19,9 & \\
\hline Secundaria incom./comp. & 58,9 & 59,6 & \\
\hline Técnica/Uni incom./comp. & 16,6 & 20,5 & \\
\hline Ingreso (\% dólares) & & & 0,483 \\
\hline Menos 350 & 4,0 & 4,0 & \\
\hline Entre $351-600$ & 56,3 & 62,9 & \\
\hline Entre $601-750$ & 39,7 & 33,1 & \\
\hline Vivienda $(\%)$ & & & 0,543 \\
\hline Propia & 52,3 & 53,3 & \\
\hline Arrendada & 23,2 & 27,2 & \\
\hline Allegado & 24,5 & 20,0 & \\
\hline
\end{tabular}

Los trabajadores de este estudio representan una población laboral joven con alrededor de una media de 36 años, lo que se corresponde con la capacidad para sobrellevar el esfuerzo que significa la realización de trabajo físico en la actividad laboral que desarrollan, observándose una edad mínima de 18 años y una máxima de 64 años (Tabla 2). La altura de los trabajadores es bastante similar entre los grupos, alcanzando un promedio de $171,9 \pm 6,8$ centímetros, al igual que el peso corporal con una media de $80,7 \pm 11,4$. Al considerar la variable IMC de manera 
Tabla 4.

Características laborales según grupo casos y grupo controles.

\begin{tabular}{|c|c|c|c|}
\hline & Casos (n: 151) & Controles ( $n: 151)$ & valor $p$ \\
\hline Rubro de la empresa (\%) & & & 0,287 \\
\hline Manufactura & 41,7 & 35,1 & \\
\hline Logistica & 58,3 & 64,9 & \\
\hline $\begin{array}{l}N^{\circ} \text { trabajadores totales, } \\
\text { mediana }\left(p_{25}-p_{75}\right)\end{array}$ & $110(50-220)$ & $100(50-225)$ & 0,357 \\
\hline Jornada Prevencionista (\%) & & & 0,353 \\
\hline Tiempo completo & 53,6 & 59,6 & \\
\hline Tiempo parcial & 46,4 & 40,4 & \\
\hline $\begin{array}{l}\text { Antigüedad laboral, } \\
\text { mediana meses }\left(\mathrm{p}_{25}-\mathrm{p}_{75}\right)\end{array}$ & $35(14-72)$ & $36(24-96)$ & 0,489 \\
\hline $\mathrm{N}^{\circ}$ horas trabajo semanal & & & 0,130 \\
\hline 45 horas & 86,1 & 78,8 & \\
\hline 48 horas & 13,9 & 21,2 & \\
\hline $\mathrm{N}^{\circ}$ trabajadores en $\mathrm{PT}(\%)$ & & & 0,003 \\
\hline 1 a 2 trabajadores & 30,5 & 47,7 & \\
\hline 3 o más trabajadores & 69,5 & 52,3 & \\
\hline \multicolumn{2}{|c|}{ Programas de prevención actualizado (\%) } & & 0,032 \\
\hline No & 60,3 & 49,0 & \\
\hline Si & 39,7 & 51,0 & \\
\hline Uso faja lumbar (\%) & & & $<0,001$ \\
\hline No & 78,8 & 60,9 & \\
\hline Si & 21,2 & 39,1 & \\
\hline
\end{tabular}

Test exacto Fisher, valor $p<0,05$, Test mediana, valor $p<0,05$

continua, la media entre los grupos también se observa similar presentando un promedio de 27,26 $\pm 3,21$. Cabe mencionar que el IMC evaluado de manera continua no muestra diferencias estadísticamente significativas; sin embargo, al utilizar la categorización propuesta por la $\mathrm{OMS}^{15}$ es posible detectar diferencias entre ambos grupos. La opción de utilizarlo de esta manera se basa en el supuesto de que los trabajadores que se encuentran en condición de sobrepeso y obeso puedan ver aumentado su riesgo de desarrollar dolor lumbar. Sobre un 60\% de los sujetos reporta ser fumador ocasional o permanente y sólo alrededor de un 20\% declara no realizar ninguna actividad física fuera del trabajo.

En relación a las características sociodemográficas (Tabla 3), los montos de ingreso entre los grupos de casos y controles se distribuyen de manera similar; en relación a esto, alrededor de un 60\% de los trabajadores reporta un ingreso menor a US 600, tanto en el grupo de casos como de controles. Finalmente, en la variable "vivienda" sobre un 50\% predomina la categoria de vivienda propia en ambos grupos. El nivel socioeconómico coincide con el grupo D y E relacionado con estudios que clasifican la población chilena ${ }^{16}$ representando, dentro de los trabajadores formales, aquellos con los niveles sociales, económicos y educacionales más inferiores de acuerdo a esta clasificación.

Las características laborales (Tabla 4) de los grupos estudiados muestra que los trabajadores pertenecen a 135 empresas diferentes y provenientes, principalmente, del rubro logístico. Este rubro incluye a empresas dedicadas a la distribución, embalaje y almacenamiento de materiales. Por su parte, las empresas del rubro manufacturero alcanzan un 41,7\% para el grupo de casos y 35,1\% para el grupo de controles. La mediana de trabajadores por empresa es similar en ambos grupos, alcanzando aproximadamente 100 trabajadores; en términos generales, las empresas contaban con un mínimo de 2 y un máximo de 9771 trabajadores.

Todas las empresas reportaron contar con un profesional prevencionista para el apoyo en materia de riesgo laboral, de las cuales sobre un 50\% cuentan con un profesional prevencionista a tiempo
Tabla 5.

Características psicosociales en el trabajo según grupo casos y grupo controles.

\begin{tabular}{|c|c|c|c|}
\hline & Casos (n: 151) & Controles (n: 151) & valor $p$ \\
\hline Exigencias psicológicas (\%) & & & $<0,001$ \\
\hline baja & 29,8 & 61,6 & \\
\hline media & 32,5 & 16,6 & \\
\hline alta & 37,8 & 21,9 & \\
\hline \multicolumn{3}{|c|}{ Autonomía y desarrollo habilidades } & $<0,001$ \\
\hline baja & 28,5 & 41,7 & \\
\hline media & 26,5 & 38,4 & \\
\hline alta & 45,0 & 19,9 & \\
\hline \multicolumn{3}{|c|}{ Apoyo social y calidad de liderazgo (\%) } & $<0,001$ \\
\hline baja & 19,2 & 41,7 & \\
\hline media & 37,1 & 32,5 & \\
\hline alta & 43,7 & 25,8 & \\
\hline \multicolumn{3}{|l|}{ Compensaciones (\%) } & $<0,001$ \\
\hline baja & 21,2 & 22,5 & \\
\hline media & 37,1 & 57,6 & \\
\hline alta & 41,7 & 19,9 & \\
\hline \multicolumn{3}{|l|}{ Doble presencia $(\%$} & $<0,001)$ \\
\hline baja & 25,8 & 29,1 & \\
\hline media & 29,1 & 35,1 & \\
\hline alta & 45,0 & 35,8 & \\
\hline
\end{tabular}

Test exacto Fisher, valor $p<0,05$.

completo en ambos grupos. Esto coincide con la exigencia legal de que empresas por sobre los 100 trabajadores deben contar con un prevencionista a tiempo completo.

En ambos grupos se estima por sobre el 50\% los puestos de trabajo con tres o más de trabajadores. La relación contractual formal con la empresa fluctúa entre las 45 y 48 horas de trabajo semanal, predominando los contratos de 45 horas semanales. Se observó, además, que la antigüedad laboral de los trabajadores en la misma empresa alcanza una mediana no superior a los 36 meses, lo que puede ser reflejo de la alta movilidad de los trabajadores entre empresas del rubro.

Con respecto a capacitación relacionada a la prevención de riesgo para la manipulación manual de cargas en los últimos 6 meses, sólo el 39\% de los trabajadores del grupo de casos reportaron haber recibido capacitaciones en este tema; por su parte, los controles alcanzaron el 50\%. El uso de faja lumbar, como equipo de protección individual, lo reporta sólo un 21,2\% de los trabajadores de casos y un $39,1 \%$ del grupo de controles; ambos grupos revelan el uso infrecuente de este dispositivo.

Según el grupo de casos y grupos de controles, las características psicosociales en el trabajo tales como exigencias psicológicas, autonomía y desarrollo de habilidades, apoyo social y calidad de liderazgo en la empresa, compensaciones y doble presencia (Tabla 5) muestran diferencias importantes cuando se analizan según las categorías baja, media y alta exposición. En el grupo de casos, la categoría "exposición alta" de las cinco dimensiones medidas, presentan proporciones de trabajadores superiores que el resto de las categorías. Por su parte, en la categoría de "exposición baja”, los controles presentan valores superiores que en los casos.

A partir de una regresión logística sin ajustar, se evaluó la asociación entre niveles de MMC con la discapacidad laboral por dolor lumbar (Tabla 6). Al evaluar la variable dummy MMC en sus distintos niveles de exposición se observa un incremento de la Odd Ratio (OR) entre las categorías (la categoría de referencia corresponde a "baja exposición”). El resultado muestra que la OR es 1,85 (IC95\%: 0,78-4,40) en 
Tabla 6.

Exposición a MMC y discapacidad por dolor lumbar, análisis no ajustado.

\begin{tabular}{lccll} 
& $\begin{array}{c}\text { OR } \\
\text { (cruda) }\end{array}$ & IC 95\% & $\begin{array}{l}\text { valor } p \\
\text { homogeneidad }\end{array}$ & $\begin{array}{l}\text { valor } p \\
\text { tendencia }\end{array}$ \\
\hline Exposición a MMC & & & $<0,001$ & $<0,001$ \\
Baja & 1,0 & & & \\
Moderada & 1,85 & $0,78-4,40$ & & \\
Alta y muy alta & 3,65 & $1,59-8,36$ & &
\end{tabular}

Tabla 7.

Modelos Regresión Logística Múltiple. Asociación entre manipulación manual de cargas y la discapacidad laboral por dolor lumbar, modelos ajustados.

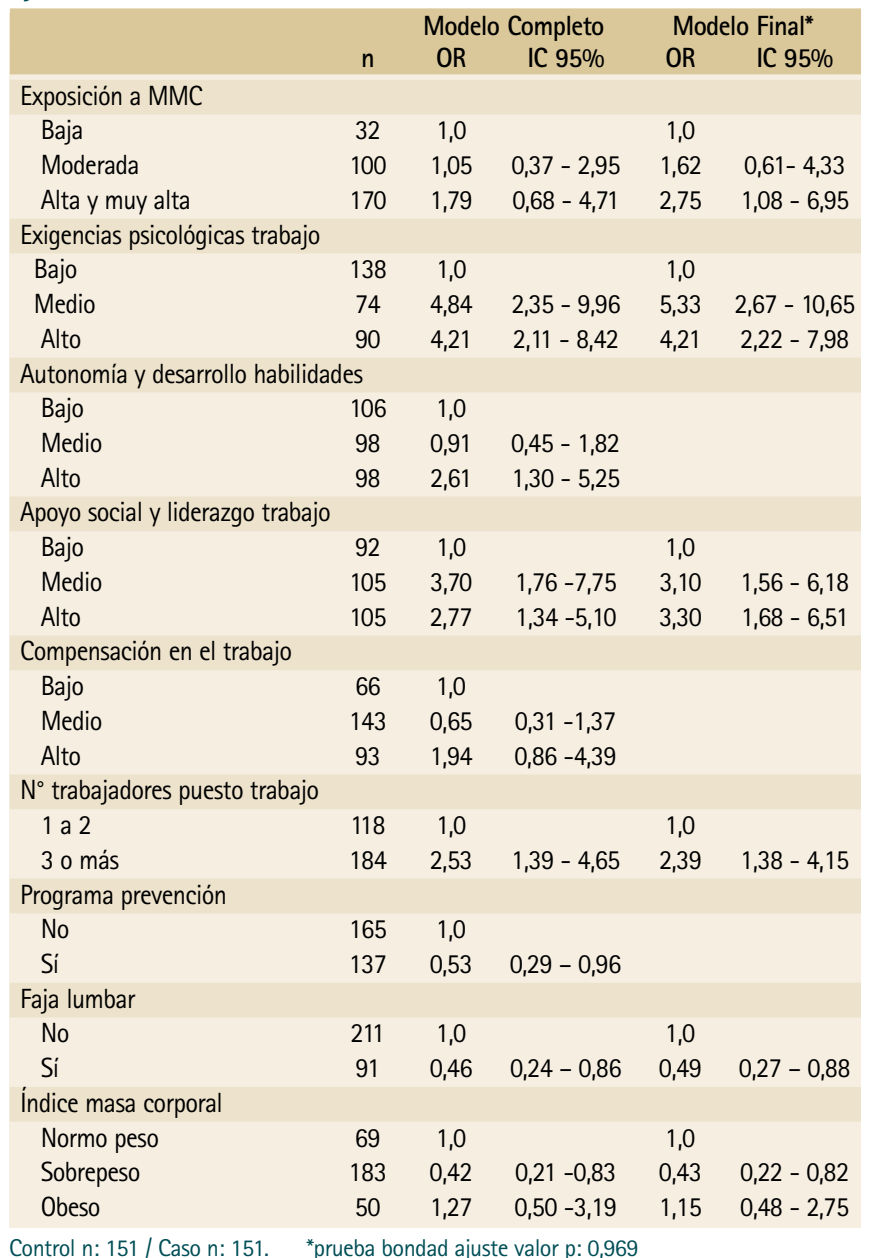

la exposición moderada y en la exposición alta y muy alta la OR es 3,65 (IC95\%: 1,59-8,36). La variabilidad del estimador de la categoría alta y muy alta es bastante similar, con amplitudes de los intervalos de confianza de 7,39 y 7,13 respectivamente. Destaca el valor del estimador en la categoría alta y muy alta comparado con la categoría moderada, superando el doble el valor del OR.

A partir de la información obtenida, se ajusta un modelo de regresión logística múltiple que incluye a todas las variables que alcanzaron la significancia estadística en los análisis de asociación, modelo que contiene cuatro variables psicosociales, tres variables relacionadas con la organización del trabajo y una variable antropométrica (Tabla 7). Este Modelo Final proporciona un valor del estimador OR de 1,62 (IC95\%: 0,61-4,33) para la categoría moderada de exposición a MMC y una OR de 2,75 (IC95\%: 1,08-6,95) para la categoría alta y muy alta, ambas en relación a la categoría de exposición baja, a pesar de no mejorar la precisión de la estimación con respecto a los modelos previos si se mejora en relación al modelo crudo.

Las covariables incluidas en el modelo y que permiten controlar el efecto de la MMC sobre la discapacidad por dolor lumbar son, en primer lugar, las exigencias psicológicas referidas al volumen del trabajo con relación al tiempo que se dispone para llevarlo a cabo. Incluyen, además, las exigencias emocionales, cognitivas y sensoriales que suponen el desarrollo del trabajo, particularmente importantes en trabajadores expuestos a exigencias por rendimiento. En segundo lugar, el factor referido a la falta de apoyo social y calidad de liderazgo se refiere a lo que las teorías sobre riesgos psicosociales han denominado las relaciones sociales en el trabajo. Para que éstas sean saludables el trabajo debe ofrecer posibilidades de relacionarse y de recibir ayuda, los puestos de trabajo deben estar bien definidos y sin exigir tareas contrarias a nuestros valores, se debe disponer de la información adecuada para desempeñarlos, y los mandos deben contar con procedimientos y habilidades para gestionar equipos humanos. Esta situación se da con frecuencia en puestos de trabajo en que se realiza MMC, dado que se encuentran permanentemente sometidos a las disposiciones de los superiores jerárquicos dentro de la organización.

El ajuste por el número de trabajadores en el puesto de trabajo se relaciona principalmente con compartir las exigencias físicas del trabajo y secundariamente con el apoyo de pares. Esta situación se da con frecuencia en puestos de trabajo con MMC debido a que, como lo demuestra este estudio, se realiza preferentemente entre dos o más personas. Por su parte, el uso de faja lumbar constituye un equipo de protección para lesiones de columna lumbar, tradicionalmente indicado en trabajos de MMC. Así como el factor individual considerado en el modelo contempla el índice de masa corporal, que puede constituir un factor que determine una mayor vulnerabilidad del trabajador sometido a esfuerzos físicos, debido a que se suman las cargas externas durante la MMC con las cargas internas que provienen de la estructura corporal del trabajador.

De acuerdo a los resultados del análisis inferencial, el Modelo Final sería el que mejor evidencia la asociación entre los niveles de MMC y la discapacidad laboral por dolor lumbar, dado que es ajustado por un grupo suficiente y razonable de covariables que abordan dimensiones que han mostrado en la literatura tener influencia y que este estudio en particular ha permitido profundizar.

Asimismo, con respecto a la valoración de la tendencia entre los niveles de exposición, esta se mantiene al comparar los tres modelos presentados en la Tabla 7, por lo cual podemos comprobar una tendencia dosis respuesta.

\section{DISCUSIÓN}

Este estudio permitió comprobar la asociación entre niveles de MMC y discapacidad por dolor lumbar en trabajadores obreros en Santiago de Chile. Los hallazgos resultan similares a lo reportado por la literatura que ha revisado la relación entre carga física y dolor lumbar. Es así como en el estudio de Burdorf $^{8}$ se muestra que el efecto de la flexión de tronco, así como la rotación del tronco y maniobras de elevación en el trabajo, están significativamente asociadas con la presencia de licencias laborales, particularmente los trabajadores que presentaron alta carga física; es necesario mencionar que gran parte de la población estudiada por Burdorf era femenina. También coin- 
cide con los resultados observados en el estudio prospectivo llevado a cabo por Hoogendoorn ${ }^{17}$, quien encontró importantes relaciones entre la flexión, rotación y elevación de tronco en el trabajo con ausencias laborales por dolor lumbar.

Por su parte Murtezani ${ }^{18}$ informó que los factores individuales no influyen en la discapacidad, mientras que los factores físicos relacionados con el trabajo mostraron asociaciones sólidas. Por ejemplo, los principales factores de riesgo de las bajas por enfermedad, debido a dolor lumbar entre los trabajadores de producción, fue la flexión del tronco extrema, así como la flexión de tronco muy extrema. El estudio de Murtezani se basó solo en aspectos biomecánicos involucrados en la actividad laboral.

Los ajustes realizados por factores individuales, psicosociales y características del trabajo mostraron una disminución en la magnitud de la asociación; sin embargo, se mantuvo una tendencia exposición respuesta, observándose que el riesgo comenzó a aumentar desde los niveles más bajos de exposición. Situación similar se encontró en el estudio prospectivo de Burdorf $^{8}$, quien también identifica esta tendencia.

En relación a los factores de ajuste, los resultados comprueban que los factores psicosociales constituyen importantes aspectos relacionados con el clima laboral y apoyo de pares y superiores, aspectos que pueden ser considerados como potenciales variables que explican que el trabajador se ausente del trabajo por cursar un dolor lumbar. Es así como la variable "exigencias psicológicas" y la variable "apoyo social y liderazgo" alcanzan magnitudes de asociación superiores a lo observado para la MMC. La literatura ${ }^{9}$ le atribuye a estos factores un importante rol en determinar el dolor lumbar y la discapacidad asociada. Los hallazgos se encuentran apoyados por el planteamiento de Hoogendoorn ${ }^{17}$ quien menciona que el efecto de las características psicosociales del trabajo puede ser más pronunciado en una población que es relativamente homogénea para otros determinantes potenciales de dolor lumbar.

Es posible que la relevancia de los factores psicosociales, en el presente estudio, esté influido porque la medición de estos factores se realizó posterior a un periodo de discapacidad laboral, dado lo cual el trabajador puede manifestar percepciones más desfavorables de su ambiente laboral que aquellos trabajadores que no han pasado recientemente por tal evento. En este sentido la mayoría de las revisiones sistemáticas ${ }^{7,9,10}$ sugieren que para el estudio de estos factores se debieran ejecutar estudios de seguimiento que permitan confirmar una relación causal.

El ajuste por la variable número de trabajadores-puesto de trabajo, que se encuentra relacionada con aspectos organizacionales del trabajo, también constituye una potencial variable que explica la discapacidad laboral por dolor lumbar, pues permitiría al trabajador apoyarse en sus compañeros en caso de ausentarse del trabajo por motivos de salud. En esta variable la categoría "3 o más trabajadores" en relación a la categoria "1 a 2 trabajadores", representa un factor fuertemente asociado y superior a lo reportado por la literatura. ${ }^{9}$

El uso del equipo de protección individual, denominado faja lumbar, aparece como una variable de ajuste relevante y significativo, que da cuenta que su uso puede significar protección al daño de columna lumbar al realizar tareas de manipulación. Esto puede ser debido a que constituye un estabilizador externo para los movimientos extremos de la región lumbar supliendo la ineficiencia de la musculatura como estabilizador fisiológico. En el ensayo clínico de Oleske ${ }^{19}$ también se revela que el uso de un soporte de la espalda constituye un protector de dolor lumbar cuando se asocia a educación sanitaria. La literatura disponible ${ }^{9}$ concluye que existe una moderada evidencia en atribuir al IMC como factor determinante de dolor lumbar. Sin embargo, en este estudio, al asociar las categorías de IMC en el modelo multivariable, la condición de sobrepeso aparece como un factor protector, mientras que la categoría de obeso se observa como factor de riesgo de presentar discapacidad por dolor lumbar. En relación a lo anterior y considerando la modalidad de cálculo con que se obtiene el IMC $^{15}$ mediante lo cual no es posible discriminar si el sobrepeso es atribuible a un aumento de tejido adiposo o un aumento de tejido muscular, es posible suponer que los trabajadores que realizan manipulación manual de cargas presentan condiciones musculares adaptadas a las exigencias de la tareas, por lo cual su masa muscular es mayor, pudiendo resultar en un factor protector de las dolencias en la zona lumbar.

Dado que el peso del objeto y la frecuencia de manipulación son los factores que más influyen en la exposición medida, es posible deducir que la exposición está principalmente determinada por las particularidades del proceso productivo y que la influencia del trabajador en ella en menor. Por su parte, las características de la maniobra de manipulación varian de manera importante entre los trabajadores y entre las tareas específicas que desarrollan, dado lo cual la efectividad de las estrategias preventivas estandarizadas pueden verse cuestionadas al no considerar las particularidades de cada lugar de trabajo. Los hallazgos de este estudio contribuyen a aumentar la evidencia científica del efecto del trabajo pesado, con el propósito de mejorar el abordaje preventivo en el contexto laboral. En dos revisiones sistemáticas ${ }^{20,21}$, se plantea que, aunque la etiología del dolor lumbar sigue siendo incierta, las actividades ocupacionales han estado siempre implicadas. Sin embargo, estas revisiones no son concluyentes, dando cuenta que falta mayor evidencia, específicamente entre la asociación de este problema de salud y la actividad laboral. ${ }^{22,23}$

En el caso de Chile, las enfermedades musculoesqueléticas representadas por el dolor lumbar predominarian entre las enfermedades que producen elevado ausentismo laboral. ${ }^{24}$ Sin embargo, sólo una parte de la población laboralmente activa, que se encuentra expuesta a esfuerzo físico, están protegidos por una política de seguridad y salud laboral $^{25}$, quedando fuera de la cobertura del seguro laboral un número importante de la población. Es a través de organismos ejecutivos y fiscalizadores que se pone en práctica la prevención de riesgos laborales, con el fin de controlar la morbilidad asociada y las consecuencias en discapacidad de los trabajadores en sus años productivos. $^{9,26-28}$ A pesar de la existencia de una política pública, las estrategias para abordarlo no parecen estar mostrando resultados satisfactorios, probablemente debido a que hasta el día de hoy no se cuenta con evidencia para catalogar al dolor lumbar como una enfermedad profesional, lo que se traduce en que los esfuerzos preventivos para controlarlo no sean suficientes.

Dentro de los escasos estudios llevado a cabo en Chile, se destaca el realizado por Diaz-Ledezma ${ }^{12}$ sobre la prevalencia de dolor lumbar agudo en Chile como causa de licencia laboral y las variables asociadas, detectando que los trabajadores manuales tenían un 35\% más licencia que otro tipo de trabajadores. Dado que este estudio incluyó trabajadores del sistema público de salud, es posible suponer que la magnitud del problema sea superior en este tipo de población debido a que no se cuenta con una protección laboral especializada. Los aspectos metodológicos considerados en este estudio ofrecieron varias ventajas. La exposición a MMC fue evaluada a través de observaciones detalladas del puesto de trabajo, consideró un grupo ocupacional bastante homogéneo en cuanto a las características de la actividad de manipulación, de manera de poder estudiar la variabi- 
lidad de la exposición entre los sujetos más que recoger variabilidad entre rubros laborales. Justamente debido a que este estudio se centró en evaluar los niveles de exposición a MMC, se buscó establecer una tendencia entre la exposición y la respuesta. Esto se debió a que revisiones sistemáticas ${ }^{7,9,10}$ que han estudiado el tema muestran la exposición a MMC, principalmente de manera dicotómica, revelando estar significativamente asociada con la presencia de discapacidad por dolor lumbar; sin embargo, no se ha demostrado con suficiente evidencia una tendencia exposición-respuesta. Un aporte de este estudio es haber encontrado una tendencia estadísticamente significativa, contribuyendo de esta manera a dar mayor fuerza en la asociación estudiada.

La relevancia del instrumento de medición para cuantificar el nivel de carga física debe destacarse al comparar los resultados con otros estudios; por ejemplo, se realizó una medición mediante observación directa desde la fuente primaria, que incluía aspectos biomecánicos y del entorno físico, en contraste con otros estudios que han recolectado información de la exposición mediante autorreportes o que, exclusivamente, incluian medición biomecánica. Por ejemplo, el estudio de Murtezani ${ }^{18}$ encontró que los trabajadores con mayor riesgo de ausencias laborales por dolor lumbar eran principalmente obreros que realizaban trabajo físico pesado y recibían un alto nivel de compensación; sin embargo, se limitó al seguimiento a una sola empresa de la cual provenían todos los trabajadores del estudio y, además, con la limitante de medir la exposición sólo mediante autorreporte. Esta importante distinción coincide con lo reportado en la revisión sistemática de $\mathrm{Wai}^{7}$, que daba cuenta de problemas de comparabilidad de los estudios por los distintos abordajes metodológicos que consideraban.

Por otra parte, la literatura referente a las causas de discapacidad laboral por dolor lumbar muestran estudios que recogen información de variados grupos ocupacionales simultáneamente: por ejemplo, en el estudio de Burdorf ${ }^{8}$ fueron nueve los grupos laborales, que incluían a enfermeras, cocineros, trabajadores del transporte, terapeutas físicos y trabajadores de oficina. En la revisión de Roffey ${ }^{10}$ los estudios evaluados incluían oficios tales como oficinistas, enfermeras y trabajadores de la construcción. Dado lo anterior, la importancia de contar con información más precisa de un grupo laboral nos permite tomar decisiones más focalizadas. Además, en el estudio de Burdorf los resultados obtenidos fueron sobre la base de una muestra de alrededor de 500 individuos, de los cuales casi un 84\% eran mujeres. Este último factor puede estar determinando que las magnitudes de asociación reportadas sean superiores a otros estudios, puesto que este factor ha sido indicado como influyente en los resultados, debido a que las mujeres han mostrado un mayor riesgo de ausencias laborales por dolor lumbar. ${ }^{29,30}$

En este estudio los trabajadores no mostraron diferencias significativas en relación a la mayoría de las variables sociodemográficas; esto da cuenta de la homogeneidad entre los grupos en función de estos factores, lo que en términos del análisis estadísticos proporcionó una buena base de comparación entre el grupo de casos y de controles.

Otra ventaja, lo que también constituye una limitación, es que se limitó a población obrera, dejando fuera otros tipos laborales que también manifiestan alta incidencia de dolor lumbar. A propósito de esto se dejaron fuera variables que podrían hacer confundir la relación entre factores relacionados al trabajo y la presencia de discapacidad laboral por dolor lumbar. Por ejemplo, el rol del nivel socioeconómico constituye un elemento complejo de analizar debido a que el tipo de trabajo relacionado con el esfuerzo físico y carga física es determinante del estatus socioeconómico. Otros aspectos como el nivel de ingresos, número de personas dependientes, estado civil, que en este estudio se comportaron de manera bastante homogénea, siguen siendo objeto de estudio debido a que aún no existe certeza de su influencia. ${ }^{31}$

Factores relacionados con estilo de vida de los trabajadores, como la condición de fumador y la realización de actividad física, han sido mencionados en la literatura; sin embargo, no se ha comprobado su vinculación con la condición de tener discapacidad por dolor lumbar. ${ }^{31}$ En este estudio tampoco se observaron hallazgos significativos.

Dado el impacto preventivo que tiene el sistema de seguridad laboral chileno sobre las enfermedades relacionadas con el trabajo, es esperable que la magnitud del problema esté controlado; sin embargo, los resultados obtenidos dan cuenta de que existe un problema de salud considerable y que aparentemente tiene su raíz en el propio trabajo. Dada la evidencia que se reporta, en relación a trabajadores protegidos por un sistema de seguridad laboral, es posible extrapolar la situación a los trabajadores que no están cubiertos por un sistema de seguridad, que a pesar de que esta investigación no los consideró, es posible suponer que sus condiciones laborales y sanitarias sean aún más desfavorables.

En el marco de la ley chilena ${ }^{25}$, los esfuerzos preventivos se focalizan en la capacitación de los trabajadores en materia prevención de riesgos y la responsabilidad de ellos en minimizar los riesgos, no obstante, estos esfuerzos se ven enfrentados con aspectos organizacionales en tareas de MMC que tienen que ver más con definiciones de la empresa y de su proceso productivo. En teoría, los programas de prevención debieran proporcionar herramientas al trabajador para su autocuidado, mediante la entrega de información de los riesgos a los cuales se enfrenta y la forma de abordarlo durante el proceso productivo, así como contribuir a mejorar las técnicas para la manipulación de cargas. Se observó que gran parte de los trabajadores con discapacidad no habían recibido capacitación en los últimos seis meses, lo que permite suponer que el fenómeno es subestimado en su magnitud y en sus consecuencias por parte de la organización.

El uso de equipos de protección individual han sido tradicionalmente vinculado a la prevención de lesión lumbar; sin embargo, este dispositivo sigue siendo controversial en la literatura ${ }^{19}$, su principal valor preventivo es en las recurrencias de dolor lumbar, sobre todo si está asociado a educación. Aparentemente su eficacia se relaciona con proporcionar una estabilidad biomecánica de la columna, pero no han sido considerados los efectos secundarios de su uso prolongado, como la disminución de la actividad muscular de la zona abdominal y lumbar, lo cual deben ser vistos en las instrucciones de uso. ${ }^{32} \mathrm{~A}$ pesar de que este estudio muestra que su uso es más bien infrecuente, aun así revela ser un factor protector en la muestra de trabajadores. La literatura ha mostrado que factores organizacionales de la empresa están vinculados con la probabilidad de desarrollar discapacidad por dolor lumbar. ${ }^{7,10}$ Dado los resultados obtenidos, trabajadores que comparten el puesto de trabajo con un mayor número de personas presentan una mayor frecuencia de ausentarse al trabajo por presentar dolor lumbar. Se pudiera pensar que el trabajo pesado, al ser compartido entre más personas, disminuye la sobrecarga y con ello la probabilidad de daño; sin embargo, este estudio muestra que, al contar con mayor apoyo de pares y, en definitiva, de la organización, es posible ausentarse del trabajo por razones médicas, probablemente debido que al contar con más trabajadores por puesto de trabajo existe la posibilidad de reemplazarse, sin que el proceso productivo se vea muy perjudicado. Esto significaría que la presencia 
de un mayor número de trabajadores por puesto de trabajo está más vinculado al proceso productivo que a una disminución de la exposición laboral de los trabajadores, evidenciando que el sistema de seguridad laboral no está interviniendo a favor de los trabajadores. Está bastante descrito en la literatura ${ }^{6,33-35}$ la influencia de los factores psicosociales en los desórdenes musculoesqueléticos, particularmente el bajo soporte social, las tareas monótonas, el estrés, la baja satisfacción y habilidad percibida para el trabajo, como factores de riesgo de dolor lumbar; sin embargo, aún no existe evidencia de la contribución específica de cada uno de ellos. ${ }^{36}$ En este estudio, cuatro de las cinco dimensiones psicosociales evaluadas presentaron diferencias significativas entre los trabajadores que presentaban discapacidad por dolor lumbar y en aquellos que no lo presentaban. Resulta interesante que el autorreporte de los casos dio cuenta, principalmente, de condiciones psicosociales desfavorables para salud, a diferencia de los controles en que predominó el autorreporte de condiciones menos desfavorables, es posible suponer que los trabajadores afectados por un problema de salud y que, a su vez, relacionen al trabajo como causa muestren una tendencia a identificar los aspectos negativos de la organización.

Estudios longitudinales vinculados con factores psicosociales y su influencia en ausencias laborales por dolor lumbar han identificado las exigencias psicológicas, el bajo apoyo social y la baja satisfacción en el trabajo como más relevantes que la falta de autonomía o control. ${ }^{37}$ Considerando la tarea laboral que desarrollan los trabajadores de este estudio y que se basa esencialmente en trabajo físico, con bajos niveles de responsabilidad en la organización, es posible que la influencia de algunos factores psicosociales tenga una mayor influencia que otros, sobre todo si se compara con trabajos que involucran principalmente exigencias cognitivas. En este sentido aparecen relevantes el apoyo social y la calidad del liderazgo, que se relacionan con un bajo soporte de los pares y supervisores. Otro elemento que aparece repetidamente reportado es la exigencia psicológica, en el sentido de la influencia de la presión del tiempo, el nivel de concentración necesario y la dependencia de otros para completar la tarea. Entre las limitaciones de la presente investigación está no haber tomado en cuenta en el diseño la variable empresa, que puede resultar importante en relación a la ausencia laboral por dolor lumbar, dada la existencia de diferentes culturas organizacionales entre las empresas, a pesar de ser de rubros similares. Para determinar la influencia de la empresa, la realización de un análisis multinivel sería necesario, en el cual diferentes niveles jerárquicos, por ejemplo, nivel individuo y nivel empresa, pueden ser tomados en cuenta y medir la influencia de las variables medidas simultáneamente; sin embargo, las unidades de observación en este estudio no fueron agrupadas en un nivel jerárquico superior. Esto se debió fundamentalmente por la forma como se planificó la obtención de la unidad de observación, captando casos incidentes independientemente de la empresa de la cual provenían.

Este estudio fue susceptible a posibles sesgos descritos para estudios de caso control, pese a los esfuerzos puestos para reducirlos. Un control riguroso de sesgos se realizó desde la etapa del diseño hasta la etapa del análisis, lo cual fue fundamental para que se obtuvieran resultados confiables. De particular preocupación fue el sesgo de selección por las características observacionales del estudio y el sesgo de recuerdo, ya que se recogieron los datos de los trabajadores que recientemente habian tenido un episodio de discapacidad por dolor lumbar. En cuanto al sesgo de selección, la estrategia de este estudio de utilizar casos incidentes, en otras palabras, casos nuevos que fueron apareciendo hasta completar el tamaño necesario de la muestra, colaboró en este sentido. Además, se optó por una definición precisa de la condición de caso, mediante la comprobación oficial, en cada uno de ellos, de estar cursando una licencia médica por dolor lumbar. Por su parte, los controles fueron seleccionados mediante un muestreo probabilístico secuencial sin reemplazo, dentro de una base de estudio bien definida, permitiendo equilibrar el efecto de la exposición en ellos. Por último, la tasa de participación en este estudio fue bastante aceptable, con un rechazo que no superó el cinco por ciento en ambos grupos, a pesar de tener que interferir en el proceso productivo del ambiente laboral para realizar las mediciones respectivas. Esto se logró al incluir acciones personalizadas y beneficios directos sobre los participantes del estudio, lo que contribuyó a que se lograran excelentes tasas de participación y respuestas. El sesgo de recuerdo fue limitado; en esto contribuyó la estandarización y protocolización de los instrumentos de medición, mejorando con ello la confianza de sus resultados; además, dado que las evaluaciones se realizaron directamente en el puesto del trabajador, supone ventajas sobre otras modalidades de evaluación indirectas.

Finalmente, dado que se consideró el uso de criterios de inclusión, se pudo controlar, en parte, la presencia de potenciales confusores, contribuyendo a una clara definición de los sujetos de estudio. Adicionalmente, la estratificación y ajuste respectivo por potenciales variables confusoras se realizó en el proceso de análisis estadístico.

Dada que la naturaleza de los estudios de caso control puede limitar su capacidad para establecer vías etiológicas, aún así, los resultados de este estudio fundamentan claramente el origen multifactorial de la condición. Este estudio confirma la evidencia sobre la manipulación manual de carga como factor de riesgo, ajustado por características psicosociales, organizacionales e individuales, dado lo cual este estudio no es compatible con la noción que la discapacidad por dolor lumbar sea producto exclusivo de la manipulación manual de cargas en el puesto de trabajo.

En conclusión, dada la existencia de riesgo ocupacional para la discapacidad transitoria por dolor lumbar en trabajadores que manipulan cargas, es también cierto que es altamente prevenible por los factores modificables que intervienen, incluyendo la alta carga de trabajo físico y también la alta presión psicosocial. Resultados tales como días de actividad restringida, discapacidad a largo plazo y utilización de servicios salud, entre otros, podrían ser abordado por una mayor especificidad de las recomendaciones técnicas y por políticas públicas que aborden más efectivamente las enfermedades musculoesqueléticas, que tienen su origen o agravamiento en el trabajo realizado, incluyendo no sólo a los trabajadores protegidos actualmente por un sistema de seguridad laboral sino también a aquellos que no lo están.

A partir de esta investigación surgen una serie de nuevas interrogantes que serian interesantes de poder contrastar con investigaciones futuras. La primera de ellas dice relación con la valoración de los sistemas de vigilancia epidemiológica en trabajadores con perfiles de riesgo ocupacional particulares; segundo, la efectividad de los programas de prevención de riesgos laborales en el contexto de la ley chilena, incluyendo la efectividad de las técnicas de manipulación de cargas y de la prevención; por ejemplo, la acomodación de los puestos de trabajo y la gestión del dolor lumbar. Lo tercero, es la evaluación de costo-efectividad de los sistemas de mecanización y automatización de los procesos en la reducción de la morbilidad musculoesquelética. Lo cuarto, la influencia de las expectativas de los trabajadores, del ritmo, cantidad de trabajo y participación; y, finalmente, impacto de los incentivos por producción en la salud de los trabajadores que hacen trabajo físico. 
1. Prevention of occupational diseases [on line]. Geneva: International Labour Organization; 2013 [cited 9 oct 2012]. Available from: http://www.lo.org/ wcmsp5/groups/public/ed_protect/protrav/safework/documents/publication/ wcms_208226.pdf.

2. Punnett $L$, Pruss-Utun $A$, Nelson D, Fingerhut $M$, Leigh J, Tak S, et al. Estimating the global burden of low back pain attributable to combined occupational exposures. Am J Ind Med. 2005;48(6):459-69.

3. Rossignol $M$, Rozenberg $S$, Leclerc A. Epidemiology of low back pain: what's new? Joint Bone Spine.2009; 76(6):608-13.

4. Santos A, Bredemeier M, Rosa $K$, Amantea V, Xavier R. Impact on the Quality of Life of an Educational Program for the Prevention of Work-Related Musculoskeletal Disorders: a randomized controlled trial. BMC Public Health. 2011;11:60.

5. Woolf A, Vos T, March L. How to measure the impact of musculoskeletal conditions. Best Pract Res Cl Rh. 2010;24(6):723-32.

6. Musculoskeletal disorders and the workplace: Low back and upper extremities [on line]. Washington: Education National Research Council-Institute of Medicine; 2001 [cited 9 oct 2012]. Available from: http://books.nap.edu/ catalog/10032.html.

7. Wai E, Roffey D, Bishop P, Kwon B, Dagenais S. Causal assessment of occupational lifting and low back pain: results of a systematic review. Spine J. 2010;10(6):554-566.

8. Burdorf $A$, Jansen J. Predicting the long term course of low back pain and its consequences for sickness absence and associated work disability. Occup Environ Med. 2006;63(8):522-529.

9. Steenstra I, Verbeek J, Heymans M, Bongers P. Prognostic factors for duration of sick leave in patients sick listed with acute low back pain: a systematic review of the literature. Occup Environ Med. 2005;62(12):851-860.

10. Roffey D, Wai E, Bishop P, Kwon B, Dagenais $S$. Causal assessment of workplace manual handling or assisting patients and low back pain: results of a systematic review. Spine J. 2010;10(7):639-651.

11. Bakker E, Verhagen A, van Trijffel E, Lucas C, Koes B. Spinal mechanical load as a risk factor for low back pain: a systematic review of prospective cohort studies. Spine. 2009;34(8):281-293.

12. Díaz-Ledezma C, Urrutia J, Romeo J, Chelen A, Gonzalez-Wilhelm L, Lavarello C. Factors associated with variability in length of sick leave because of acute low back pain in Chile. Spine J. 2009;9(12):1010-1015.

13. Guia Técnica para la Evaluación y Control de los riesgos asociados al Manejo o Manipulación manual de Carga [en línea]. Santiago: Ministerio del Trabajo y Previsión Social de Chile; 2008 [citado 9 oct 2012]. Disponible en: http://www. dt.gob. cl/1601/article-95553.html

14. Cuestionario de Evaluación de Riesgos Psicosociales en el Trabajo, SUSESOISTAS 21 [en linea]. Santiago: Superintendencia de Seguridad Social Ministerio del Trabajo Chile; 2009 [citado 9 oct 2012]. Disponible en: http://www.suseso. $\mathrm{cl} /$ cuestionario-de-evaluacion-de-riesgos-psicosociales-en-el-trabajo-susesoistas-21/

15. Preventing and Managing the Global Epidemic [on line]. Geneve: Organization World Health; 2000 [citado 9 oct 2012]. Disponible en: http://www.who.int/ nutrition/publications/obesity/WHO_TRS_894/en

16. Mapa socioeconómico de Chile [en línea]. Santiago: Adimark; 2011 [citado 9 oct 2012]. Disponible en: http://www.adimark.cl/medios/estudios/mapa_socio economico_de_chile.pdf.

17. Hoogendoorn $W$, de Vet $H$, Ariens $G$, van Mechelen W, Bouter L. High physical work load and low job satisfaction increase the risk of sickness absence due to low back pain: results of a prospective cohort study. Occup Environ Med. 2002;59:232-328
18. Murtezani A, Hundozi $H$, Orovcanec N, Berisha M, Meka V. Low back pain predict sickness absence among power plant workers. Indian J Occup Environ Med. 2010; 14(2):49-53.

19. Oleske $D$, Lavender $S$, Andersson G, Kwasny M. Are back supports plus education more effective than education alone in promoting recovery from low back pain?: Results from a randomized clinical trial. Spine. 2007;32(19):2050-2057.

20. Kuijer W, Groothoff J, Brouwer S, Geertzen J, Dijkstra P. Prediction of sickness absence in patients with chronic low back pain: a systematic review. J Occup Rehabil. 2006;16(3):439-467.

21. Hayden J, Chou R, Hogg-Johnson S, Bombardier C. Systematic reviews of low back pain prognosis had variable methods and results: guidance for future prognosis reviews. J Clin Epidemiol. 2009;62(8):781-796.

22. Williams $R$, Westmorland $M$, Lin $C$, Schmuck $G$, Creen M. Effectiveness of workplace rehabilitation interventions in the treatment of work-related low back pain: a systematic review. Disabil Rehabil. 2007;29(8):607-624.

23. Heitz $C$, Hilfiker $R$, Bachmann $L$, Joronen $H$, Lorenz $T$, Uebelhart $D$, et al. Comparison of risk factors predicting return to work between patients with subacute and chronic non-specific low back pain: systematic review. Eur Spine J. 2009;18(12):1829-1835.

24. Concha M. Enfermedades Profesionales: Una Aproximación a su Frecuencia. Cienc Trab. 2007; 9(25):117-120.

25. Ley 16.744; establece normas sobre accidentes del trabajo y enfermedades profesionales del Ministerio de Trabajo y Previsión Social (23 ene 1968). Ley Chile [en línea]. [citado 9 oct 2012]. Disponible en: http://www.leychile.cl/ Navegar?idNorma $=28650$

26. Kent $P$, Keating J. The epidemiology of low back pain in primary care. Chiropr Osteopat. 2005;13:13.

27. Thelin $A$, Holmberg $S$, Thelin N. Functioning in neck and low back pain from a 12-year perspective: a prospective population-based study. J Rehabil Med. 2008:40(7):555-561.

28. Lidgren L. The bone and joint decade 2000-2010. Bull World Health Organ. 2003;81(9):629.

29. Hoy D, Brooks $P$, Blyth F, Buchbinder R. The Epidemiology of low back pain. Best Pract Res Cl Rh. 2010;24(6):769-781.

30. Kopec J, Sayre E, Esdaile J. Predictors of back pain in a general population cohort. Spine. 2004;29(1):70-77.

31. Fransen $M$, Woodward $M$, Norton $R$, Coggan $C$, Dawe $M$, Sheridan N. Risk factors associated with the transition from acute to chronic occupational back pain. Spine. 2002;27(1):92-98.

32. Hoogendoorn $W$, Bongers $P$, de Vet $H$, Douwes $M$, Koes $B$, Miedema $M$, et al. Flexion and rotation of the trunk and lifting at work are risk factors for low back pain: results of a prospective cohort study. Spine. 2000;25(23):3087-3092.

33. Marras W, Cutlip R, Burt S, Waters T. National occupational research agenda (NORA) future directions in occupational musculoskeletal disorder health research. Appl Ergon. 2009;40(1):15-22.

34. Hoogendoorn $W$, van Poppel $M$, Bongers $P$, Koes B, Bouter L. Systematic review of psychosocial factors at work and private life as risk factors for back pain. Spine. 2000;25(16):2114-2125.

35. Linton S. Occupational psychological factors increase the risk for back pain: a systematic review. J Occup Rehabil. 2001;11(1):53-66.

36. Ramond $A$, Bouton $C$, Richard I, Roquelaure $Y$, Baufreton $C$, Legrand $E$, et al. Psychosocial risk factors for chronic low back pain in primary care--a systematic review. Fam Pract. 2011;28(1):12-21.

37. Nahit $E_{1}$ Hunt I, Lunt $M$, Dunn $G$, Silman A, Macfarlane GJ. Effects of psychosocial and individual psychological factors on the onset of musculoskeletal pain: common and site-specific effects. Ann rheum dis. 2003;62(8):755-760. 